SEPSIS syndrome (SS) is associated with depressed PAF acetylhydrolase, the enzyme responsible for the degradation of platelet activating factor. PAF acetylhydrolase is in a large part produced by macrophages, whose inadequate activation with haemophagocyto$s$ is is frequent in patients with SS.

The aim of this study was to test the hypothesis that PAF acetylhydrolase levels could be affected in these critically ill patients, because of the large amounts produced by activated macrophages in vitro and in vivo in animal models.

The levels of serum PAF acetylhydrolase were assessed in 90 SS patients, who were divided into three groups: patients with $(n=34)$ or without haemophagocytosis $(n=31)$, and patients without thrombocytopenia $(n=25)$ who were used as a control group. The number of organ dysfunctions was matched between patients with haemophagocytosis and controls. Normal reference values were obtained in 59 randomly selected blood donors.

Circulating levels of PAF acetylhydrolase were significantly $(p=0.0001)$ decreased in patients with SS $(57 \pm 3 \mathrm{nnol} / \mathrm{ml} / \mathrm{min}, n=90)$ when compared with healthy subjects $(69 \pm 3 \mathrm{nmol} / \mathrm{ml} / \mathrm{min}, n=59)$. PAF acetylhydrolase levels were greater in the presence of a haemophagocytos is but without statistical significance $(64.2 \pm 6.5$ vs $50.1 \pm 2.8: p=0.25)$.

Despite the fact that macrophagic activation stimulates the in vitro release of PAF acetylhydrolase, no difference was found between patients with or without haemophagocytosis. The mechanism and the role of the PAF acetylhydrolase reduction in SS patients remain to be determined.

Key words: Platelet-activating factor acetylhydrolase, Monocyte, Macrophage, Sepsis syndrome, Multiple organ dysfunction, Macrophagic activation

\section{Platelet-activating factor acetylhydrolase and haemophagocytosis in the sepsis syndrome}

\author{
Franck Trimoreau', CA, Bruno François ${ }^{2}$ \\ Arnaud Desachy ${ }^{2}$, Arnaud Besse ${ }^{1}$, Philippe Vignon ${ }^{2}$ \\ and Yves Denizot ${ }^{1 *}$
}

${ }^{1}$ Department of Haematology, ${ }^{2}$ Intensive Care Unit, Dupuytren University Hospital, Limoges, France; *Present address: EP CNRS 118, Faculty of Medicine, 2 rue Dr Marcland, 87025 Limoges, France

\footnotetext{
${ }^{\mathrm{CA}}$ Corresponding Author Tel: +33 (5) 55.05.61.80 Fax: +33 (5) 55.05.61.85 Email: gachard@unilim.fr
}

\section{Introduction}

The sepsis syndrome (SS) is a complex inflammatory response to various acute insults. ${ }^{1}$ Platelet-activating factor (PAF), one of the main mediators involved in the inflammatory response, is produced through the metabolic pathway of the phospholipase A2 from phospholipids of the cell membrane. ${ }^{2}$ PAF plays a key role in the initiation and development of various critical diseases which are associated with increased levels of phospholipase A2, including septic shock, adult respiratory distress syndrome and multiple organ failure (MOF) ${ }^{3}$ Blood concentrations of PAF are regulated by a circulating enzyme, the PAF acetylhydrolase, which converts PAF into inactive lyso-PAF ${ }^{4}$ Depressed PAF acetylhydrolase has been reported in association with severe sepsis ${ }^{5}$ and MOF. $^{3}$ This disregulation of PAF/PAF acetylhydrolase may result in an amplified inflammatory response secondary to the increased half-life of circulating PAF. Monocytes and macrophages physiologically participate in the regulation of the balance of PAF/PAF acetylhydrolase. Stimulated monocytes release a large fraction of their newly synthesised $\mathrm{PAF}^{6}$ whereas macrophages are a major source of PAF acetylhydrolase, this process being a mechanism for the resolution of the inflammatory response. ${ }^{7}$ Recently a study has demonstrated that the PAF acetylhydrolase in human plasma originates from haematopoietic lineage cells. ${ }^{8}$

We have reported the frequent association between severe SS with thrombocytopenia and haemophagocytosis. ${ }^{9}$ As a strong macrophagic differentiation occurs in these patients, ${ }^{9}$ we hypothesized that PAF acetylhydrolase levels could be affected in these SS patients, because of the large amounts produced by activated macrophages in vitro and in vivo in animal models. ${ }^{10}$ We then conducted the present study to compare the levels of serum PAF acetylhydrolase between SS patients with or without haemophagocytosis. 


\section{Patients and methods}

\section{Patients}

All aspects of the study protocol were approved by the committee on human investigations of our institution. During a 12-month period, all consecutive patients who fulfilled the following inclusion criteria were studied: the presence of (1) an SS according to Bone's criteria, ${ }^{1}$ and (2) a thrombocytopenia (platelet count $<100000 / \mathrm{mm}^{3}$ ) of undetermined origin. Patients were excluded from the study when at least one of the following criteria were present: hematological disease, previous anticancer chemotherapy, major bleeding and/or prior transfusion of any blood component, disseminated intravascular coagulation defined as the presence of a prothrombin time of less than $50 \%$ of the control value together with either a positive ethanol test or D-dimers test, and previous administration of heparin. In each patient, the severity of the acute illness at the time of admission to the intensive care unit (ICU) was assessed using the APACHE II score. ${ }^{11}$ The potential causes of the sepsis syndrome as well as the number and nature of acute organ system dysfunction were evaluated at the time of enrolment. ${ }^{12}$ Patients were considered as infected if an ongoing infection was documented at enrolment. Bacteraemia was defined by at least two positive blood cultures with the same pathogen. Focal infections were diagnosed on the basis of both clinical and microbiological findings. In each patient, a bone marrow aspirate was performed at the day of enrolment to study the cause of thrombocytopenia. It allowed us to divide patients into two subsets, according to the presence or absence of cytological findings associated with a macrophagic activation. Haemophagocytosis was assessed by cytological examination of May Grünwald Giemsa stained bone marrow smears, using a previously described protocol. ${ }^{9}$ Haemophagocytosis was diagnosed when the percentage of macrophages exceeded $2 \%$ of bone marrow nucleated cells, in conjunction with the presence of more than one haemophagocytic cell per field at $\times 200$ magnification.

During the same period, consecutive patients with Ss but no thrombocytopenia were also studied. Although the bone marrow was not studied for ethical reasons in this group, the absence of thrombocytopenia was considered as potentially predictive of the absence of haemophagocytosis. Patients in this group were matched in terms of number of organ dysfunctions, according to the ODIN score, ${ }^{12}$ with those suffering from haemophagocytosis.

\section{PAF acetylhydrolase measurement}

Serum PAF acetylhydrolase levels were measured on the day of enrolment by assessing the degradation of $\left[{ }^{3} \mathrm{H}\right]$ acetyl-PAF. ${ }^{13}$ Briefly, $1 \times 10^{5} \mathrm{dpm}$ of $\left[{ }^{3} \mathrm{H}\right]$ acetyl-
PAF, $10 \mathrm{Ci} / \mathrm{mmol}$ (NEN, Les Ulis, France), $0.1 \mathrm{mM}$ PAF (Tebu, France), HEPES buffer ( $\mathrm{pH} 7.8$ ) in a final volume of $450 \mu \mathrm{l}$, and $50 \mu \mathrm{l}$ of serum (1/50 dilution in HEPES buffer) were incubated for $15 \mathrm{~min}$ at $37^{\circ} \mathrm{C}$. The reaction was stopped with $100 \mu \mathrm{l}$ BSA $(10 \%)$ and $500 \mu \mathrm{l}$ trichloracetic acid (20\%). Samples were centrifuged $(1500 \times g, 15 \mathrm{~min})$, supernatants were mixed with $2.5 \mathrm{ml}$ of scintillation fluid (Opti-fluor, Packard, France) and counted in a liquid scintillation counter (Packard). Results were expressed as nanomoles of PAF degraded per ml of serum permin, as means of duplicate determinations. Normal reference values were established by measuring serum concentrations of PAF acetylhydrolase in 59 randomly selected healthy subjects.

\section{Statistical analysis}

Data were expressed as mean \pm SEM. Continuous variables were compared between groups using either the Mann-Whitney U-test or the Kruskall Wallis one-way analysis of variance. Qualitative variables were compared using the chi-square test, with correction for continuity when appropriate. A $p$ value of $<$ 0.05 was considered statistically significant.

\section{Results}

Ninety patients (mean age: $58 \pm 2$ years, APACHE II: 23 \pm 1 ) were enrolled into the study. Reasons for admission to the intensive care unit included multisystem trauma $(n=15)$, complicated postoperative course $(n=23)$, and severe medical illnesses $(n=52)$. Seventy-four patients (82\%) sustained a multiple organ dysfunction and 31 (34\%) died during their stay in the ICU. Sixty-five patients were diagnosed with thrombocytopenia. Megakaryocytic lineage was always normal. Macrophagic activation was identified on bone marrow smears in 34 cases (52\%). When compared with patients without macrophagic activation, the presence of a haemophagocytosis was associated with a higher number of organ dysfunctions and greater infection rate (Table 1). Patients without thrombocytopenia $(n=$ 25) had a similar number of organ dysfunctions and mortality rate, compared with those with haemophagocytosis (Table 1).

When compared with healthy subjects, serum PAF acetylhydrolase was significantly decreased in patients with SS $(57 \pm 3 \mathrm{nmol} / \mathrm{ml} / \mathrm{min}$ vs $69 \pm 3 \mathrm{nmol} /$ $\mathrm{ml} / \mathrm{min}, p=0.0001$ ) (Fig. 1). Patients sustaining multisystem trauma had significantly $(p<0.01)$ reduced serum PAF acetylhydrolase concentrations $(47 \pm 3 \mathrm{nmol} / \mathrm{ml} / \mathrm{min})$ when compared with patients with SS of medical $(58 \pm 4 \mathrm{nmol} / \mathrm{ml} / \mathrm{min})$ or surgical origin $(59 \pm 6 \mathrm{nmol} / \mathrm{ml} / \mathrm{min}$ ) (Fig. 1). In thrombocytopenic patients with haemophagocytosis, mean PAF acetylhydrolase levels were greater than those measured in patients without macrophagic activation 
Table 1. Characteristics of the different subsets of patients with sepsis syndrome

\begin{tabular}{lccc}
\hline & $\begin{array}{c}\text { Without } \\
\text { thrombocytopenia } \\
(n=25)\end{array}$ & $\begin{array}{c}\text { Thrombocytopenia } \\
\text { without } \\
\text { haemophagocytosis } \\
(n=31)\end{array}$ & $\begin{array}{c}\text { Thrombocytopenia } \\
\text { with } \\
\text { haemophagocytosis } \\
(n=34)\end{array}$ \\
\hline Age (years) & $59 \pm 3$ & $56 \pm 3$ & $60 \pm 2$ \\
Males ( $n)$ & $19(76)$ & $19(61)$ & $25(73)$ \\
APACHE II score & $23 \pm 1.4$ & $22 \pm 1.2$ & $23 \pm 1.6$ \\
Organ dysfunctions & $2.8 \pm 0.1$ & $2.0 \pm 0.2$ & $2.8 \pm 0.2^{*}$ \\
Type of organ dysfunction & $17(68)$ & $11(35)$ & $25(73)^{*}$ \\
Cardiovascular & $25(100)$ & $29(93)$ & $32(94)$ \\
Respiratory & $3(12)$ & $1(3)$ & $12(35)^{*}$ \\
Hepatic & $12(48)$ & $11(35)$ & $16(47)$ \\
Renal & $14(56)$ & $0(25)$ & $6(17)$ \\
Neurologic & 0 & $11(35)$ & $5(14)^{*}$ \\
Haematologic & $16(64)$ & $8(25)$ & $21(61)^{*}$ \\
Infections & $8(32)$ & $15(44)$ \\
Death & & & \\
\hline
\end{tabular}

${ }^{*} p<0.01$ when compared with patients without haemophagocytosis. Numbers between parentheses are percentages.

although the difference failed to reach statistical significance $(64 \pm 6 \mathrm{nmol} / \mathrm{ml} / \mathrm{min}$ vs $50 \pm 3 \mathrm{nmol} / \mathrm{ml} /$ min, $p=0.25)$. When compared with healthy subjects, patients with haemophagocytosis had similar serum PAF acetylhydrolase concentrations (64 \pm $6 \mathrm{nmol} / \mathrm{ml} / \mathrm{min}$ vs $69 \pm 3 \mathrm{nmol} / \mathrm{ml} / \mathrm{min}, p>0.05)$ whereas patients without macrophagic activation had significantly lower levels ( $50 \pm 3$ vs $69 \pm 3, p=0.001)$. SS patients with or without thrombocytopenia do not have different serum PAF acetylhydrolase levels (Fig. 1). Circulating PAF acetylhydrolase levels were not significantly increased in the presence of a bacterial infection $(51 \pm 4 \mathrm{nmol} / \mathrm{ml} / \mathrm{min}$ vs $59 \pm 4 \mathrm{nmol} / \mathrm{ml} /$ $\min , p=0.47)$ and were not predictive of fatal outcome $(58 \pm 7 \mathrm{nmol} / \mathrm{ml} / \mathrm{min}$ vs $55 \pm 4 \mathrm{nmol} / \mathrm{ml} /$ $\min , p=0.68$ ). Both number and nature of organ dysfunctions were not correlated with serum PAF

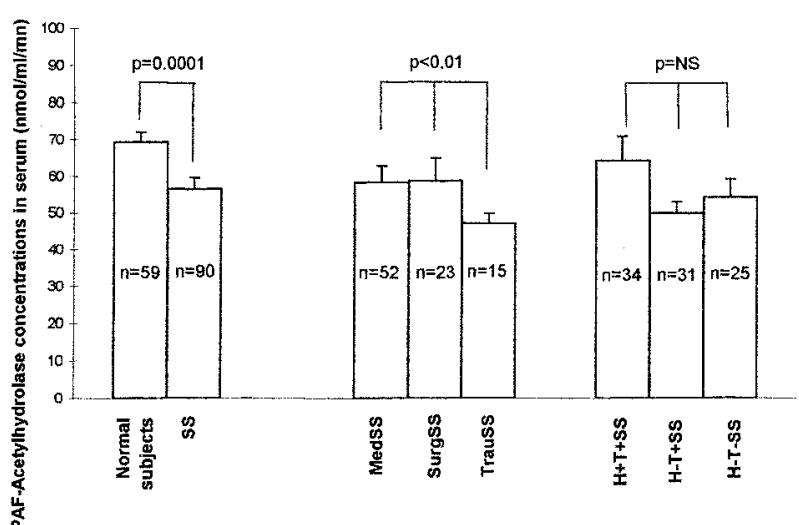

FIG. 1. Serum PAF acetylhydrolase concentrations in patients with a sepsis syndrome (SS). Data are shown for subsets of patients admitted to the intensive care unit for medical (medSS) or surgical illness (surgSS), for multisystem trauma (trauSS) and for subgroups of patients with thrombocytopenia plus haemophagocytosis $(\mathrm{H}+\mathrm{T}+\mathrm{SS})$, thrombocytopenia without haemophagocytosis $(\mathrm{H}-\mathrm{T}+\mathrm{SS})$ or patients without thrombocytopenia (H-T-SS). NS = non-significant. acetylhydrolase levels (data not shown), with the exception of neurological dysfunction which was associated with lower levels $(47 \pm 3 \mathrm{nmol} / \mathrm{ml} / \mathrm{min}$ vs $60 \pm 4 \mathrm{nmol} / \mathrm{ml} / \mathrm{min}, p=0.04)$.

\section{Discussion}

In the present study, decreased levels of PAF acetylhydrolase were found in patients with SS when compared with healthy subjects. This finding is in agreement with previously reported results in patients suffering from sepsis $^{5}$ and $\mathrm{MOF}^{3}$ Reduction of the PAF acetylhydrolase in these settings is thought to increase substantially the half-life of PAF, which in turn may amplify the inflammatory cascade in response to an acute insult, leading to MOF. ${ }^{14}$ Although lower PAF acetylhydrolase levels have been reported in patients who die from sepsis when compared with survivors, ${ }^{5}$ they were not predictive of the outcome in our patients. In addition, we did not confirm the correlation between PAF acetylhydrolase levels and the number of organ dysfunctions previously reported. ${ }^{3}$ Such a discrepancy may be explained by different study designs. In the present study, PAF acetylhydrolase levels were measured when MOF was already initiated, whereas Partrick et al. ${ }^{3}$ compared the PAF acetylhydrolase determined at admission with subsequent occurrence of organ dysfunctions during the ICU stay. Patients with SS secondary to severe multisystem trauma had lower PAF acetylhydrolase levels than those with SS of medical or surgical origin. Direct tissue injuries observed in patients sustaining severe trauma may lead to extensive cellular damage and subsequent elevated phospholipase A2 activity. Increased release of PAF by injured tissue associated with reduced levels of serum PAF acetylhydrolase might amplify organ dysfunctions in this setting when compared with SS of other origin. 
As previously reported, ${ }^{9}$ haemophagocytosis occurred frequently in thrombopenic patients with SS (52\%). When compared with patients without evidence of macrophagic activation, patients with haemophagocytosis exhibited a greater number of organ dysfunctions and a higher frequency of bacterial infections. Despite the great level of maturation of monocytes to macrophages in SS patients with haemophagocytosis, their serum PAF acetylhydrolase levels were only weakly elevated as compared with patients without haemophagocytosis or without thrombocytopenia. Moreover they were only similar to those of healthy subjects when a much greater value was expected. In SS patients with haemophagocytosis PAF acetylhydrolase production might be insufficient to resolve the inflammatory response mediated by PAF, explaining the high rate of MOF in these patients. These normal or decreased PAF acetylhydrolase levels in SS, despite a great level of maturation of monocytes to macrophages, might be due to an inactivation by free oxygen radicals of the PAF acetylhydrolase normally produced. ${ }^{15}$ In SS, these reactive oxygen species, produced by polymorphonuclear cells in response to stimulation by PAF, may play a key role in the initiation of MOF. This effect could be mediated, in part, by this inactivation of PAF acetylhydrolase, leading to an imbalance between PAF and PAF acetylhydrolase and to a default in the control of the inflammatory response.

In conclusion, our study shows that the macrophagic differentiation and activation that occurs during haemophagocytosis in SS patients is not associated with increased levels of PAF acetylhydrolase, by contrast with a usual adequate inflammatory response. This phenomenon probably reflects the disregulated character of this response, and may contribute to the mediation of organ dysfunctions in this syndrome.

\section{References}

1. Bone RC, Balk RA, Cerra FB and the ACCP/SCCM consensus conference committee. Definitions for sepsis and organ failure and guidelines for the use of innovative therapies in sepsis. Chest 1992; 101:1644-55

2. Denizot Y. Platelet-activating factor: biosynthesis, biodegradation, actions. In: von Breuchhausen F. Walter U, eds Handbook of Experimental Pharma cology, Springer-Verlag, Heidelberg, 1997: 483-506

3. Partrick DA, Moore EE, Moore FA, Biffl WL, Barnett CC. Reduced PAF acetylhydrolase activity is associated with post-injury multiple organ failure. Shock 1997; 7:170-4

4. Stafforini DM, Prescott SM, Zimmerman GA, McIntyre TM. Platelet activating factor acetylhydrolase activity in human tissues and blood cells. Lipids 1991; 26:979-85

5. Graham RM, Stephens CJ, Silvester W, Leong LLL, Sturm MJ, Taylor RR. Plasma degradation of platelet-activating factor in severely ill patients with clinical sepsis. Crit Care Med 1994; 22:204-12

6. Elstad MR, Stafforini DM, Mc Intyre TM, Prescott SM, Zimmerman GA. Platelet-activating factor acetylhydrolase increases during macrophage differentiation. J Biol Chem 1989; 264:8467-70

7. Elstad MR, Stafforini DM, Prescott SM, McIntyre TM, Zimmerman CA. Human macrophages secrete platelet activating factor acetylhydrolase. Chest 1991; 99:9S-10S

8. Asano K, Okamoto S, Fukunaga K, Shiomi T, Mori T, Iwata M, Ikeda Y, Yamaguchi K. Cellular source(s) of platelet-activating factor acetylhydrolase activity in plasma. Biochem Biophys Res Commun 1999; 261:511-14

9. Fran ois B, Trimoreau F, Vignon P, Fixe P, Praloran V, Gastine H. Thrombocytopenia in the sepsis syndrome: role of hemophagocytosis and macrophage colony stimulating factor. Am J Med 1997; 103:114-20

10. Memon RA, Fuller J, Moser AH, Feingold KR, Grunfeld C. In vivo regulation of plasma platelet-activating factor acetylhydrolase during the acute phase response. Am J Physiol 1999; 277:94-103

11. Knaus WA, Draper EA, Wagner DP, Zimmerman JE. APACHE II: a severity of disease classification system. Crit Ca re Med 1985; 13:818-29

12. Fagon JY, Chastre J, Novara A, Medioni P, Gibert C. Characterization of intensive care unit patients using a model based on the presence or absence of organ dysfunctions and/or infection: the ODIN model. Intens Care Unit 1993; 19:137-44

13. Dupuis F, Levasseur S, Jean-Louis F, Dulery C, Praloran V, Denizot Y, Michel L. Production, metabolism and effect of platelet-activating factor on the growth of the human K562 erythroid cell line. Biochim Biophys Acta 1997; 1359:241-9

14. Beal AL, Cerra FB. Multiple organ failure in the 1990s. Systemic inflammatory response and organ dysfunction. JAMA 1994; 271:226-33

15. Ambrosio G, Oriente A, Napoli C, Palumbo G, Chiarello P, Marone G, Condorelli M, ChiarelloM, Triggiani M. Oxygen radicals inhibit human plasma acetylhydrolase, the enzyme that catabolizes platelet activating factor. J Clin Invest 1994; 93:2408-16

\section{Received 9 May 2000; accepted 31 May 2000}




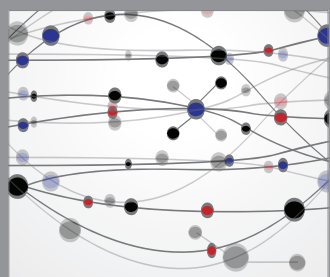

The Scientific World Journal
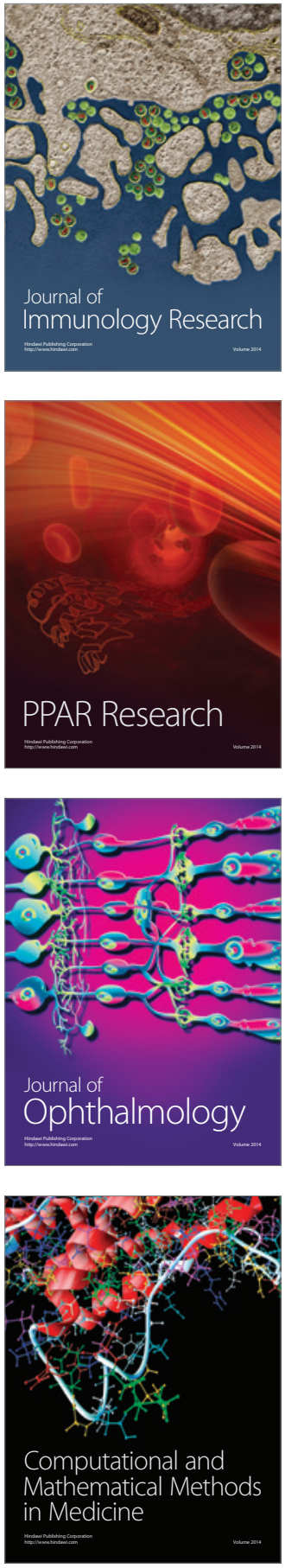

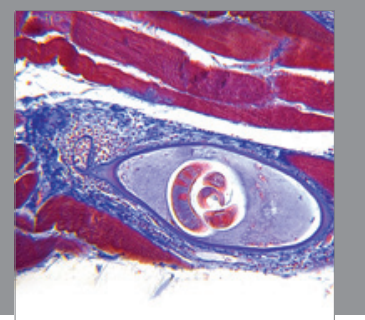

Gastroenterology

Research and Practice
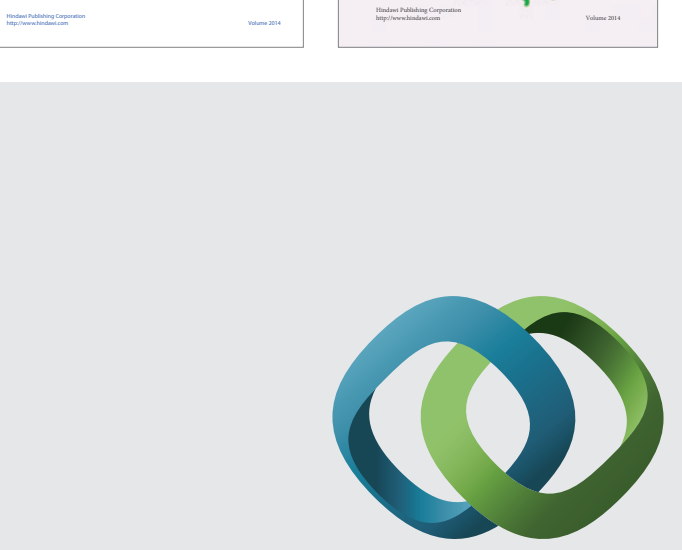

\section{Hindawi}

Submit your manuscripts at

http://www.hindawi.com
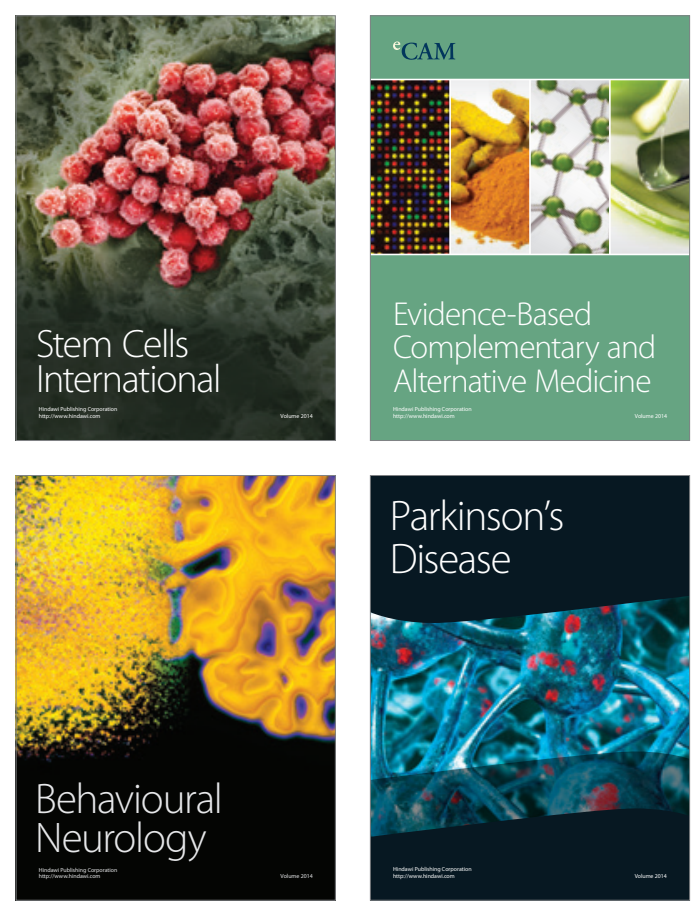

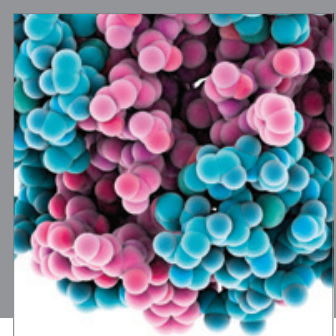

Journal of
Diabetes Research

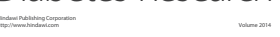

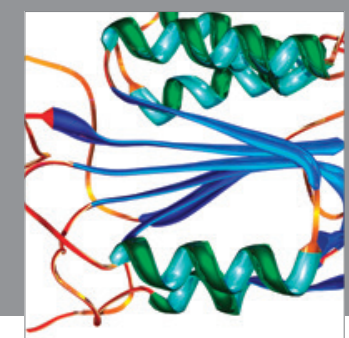

Disease Markers
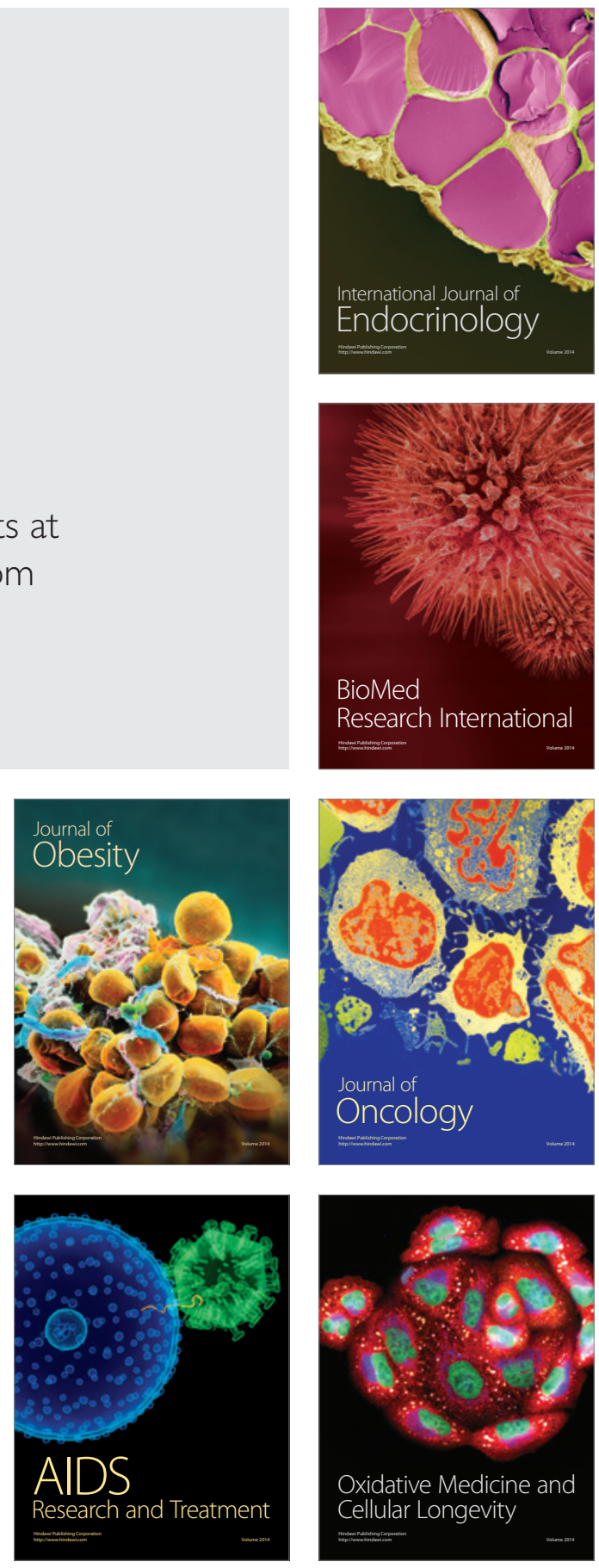